\title{
Compatibilidade de nematóides entomopatogênicos e extratos vegetais aquosos visando o controle da mosca-das-frutas Ceratitis capitata (Wiedemann) (Diptera: Tephritidae)
}

\section{Compatibility of entomopathogenic nematodes and aqueous plant extracts aiming at the control of fruit fly Ceratitis capitata (Wiedemann) (Diptera: Tephritidae)}

\author{
Cristhiane Rohde ${ }^{1 *}$; Alcides Moino Junior ${ }^{2}$; Natália Ramos Mertz ${ }^{3}$; \\ Patrícia Krupa ${ }^{4}$; Kaio Roger de Oliveira Ramalho ${ }^{5}$
}

\begin{abstract}
Resumo
A mosca-das-frutas Ceratitis capitata (Diptera: Tephritidae) tem sido controlada, principalmente, pelo método químico, que é o responsável por impactos ambientais e na saúde pública e, muitas vezes, tem sido ineficiente, devido à seleção de populações de insetos resistentes. Assim, tem surgido a necessidade de pesquisas com novas formas de controle eficazes e menos impactantes. Nesse sentido, a utilização de nematóides entomopatogênicos e extratos vegetais tem se mostrado eficiente para o controle dessa praga. No entanto, são necessários estudos para avaliar a compatibilidade entre esses métodos, visando o seu uso, em programas de manejo integrado, para essa praga. O objetivo desse trabalho foi avaliar a compatibilidade dos nematóides Steinernema carpocapsae ALL e Heterorhabditis sp. JPM4 com os extratos aquosos, preparados com vegetais secos da folha, ramo e fruto de cinamomo (Melia azedarach), folha de arruda (Ruta graveolens), gengibre (Zingiber officinales) e alho (Allium sativum) visando o controle de C. capitata. O bioensaio foi conduzido em delineamento inteiramente casualizado, com quatro repetições, por tratamento. Cada repetição foi constituída de um tubo de vidro, contendo $1 \mathrm{~mL}$ de extrato vegetal, $40 \%$ p/v e $1 \mathrm{~mL}$ de suspensão de nematóide entomopatogênico, com $1800 \mathrm{JI} / \mathrm{mL}$, para S. carpocapsae ALL e $600 \mathrm{JI} / \mathrm{mL}$, para Heterorhabditis sp. JPM4. Foi avaliada a viabilidade dos nematóides e a infectividade desses sobre larvas de $C$. capitata, após 48 e 120 horas. Verificou-se que todos os extratos reduziram a viabilidade e a infectividade de ambos os nematóides, sendo incompatíveis, após 120 horas de exposição. O nematóide Heterorhabditis sp. JPM4 foi mais sensível em relação ao S. carpocapsae ALL, pois apresentou, nas primeiras 48 horas redução na viabilidade e infectividade superior a 80 e $75 \%$, respectivamente, quando exposto a todos os extratos, exceto o de gengibre.

Palavras-chave: Steinernema carpocapsae, Heterorhabditis sp., Melia azedarach, Ruta graveolens, Allium sativum, Zingiber officinale
\end{abstract}

1 Prof ${ }^{a}$. Adjunto da Universidade Federal da Fronteira Sul, UFFS, Laranjeiras do Sul, PR. E-mail: cristhiane.rohde@uffs.edu.br

2 Prof. Associado do Dept ${ }^{\circ}$ de Entomologia, Universidade Federal de Lavras, UFLA, Campus Universitário, Lavras, MG. E-mail: alcmoino@den.ufla.br

3 Discente de Doutorado do Programa de Pós-graduação em Entomologia, UFLA, Campus Universitário, Lavras, MG. E-mail: natiramosmertz@yahoo.com.br

4 Discente de Mestrado do Programa de Pós-graduação em Produção Vegetal, Universidade Estadual do Centro-Oeste, UNICENTRO, Campus Cedeteg. Guarapuava, PR. E-mail: patyks@hotmail.com

5 Discente de graduação do curso de Agronomia, UNICENTRO, Campus Cedeteg. Guarapuava, PR. E-mail: kaiocanario@, hotmail.com

* Autor para correspondência 


\begin{abstract}
Currently, the fruit fly Ceratitis capitata (Diptera: Tephritidae) has been controlled mainly by the chemical method, which is responsible for environmental and public health impacts. It has often been ineffective due to selection of resistant insect populations. Thus, it has necessary to research new effective and less impacting control forms. In this sense, the use of entomopathogenic nematodes and plant extracts has been effective for controlling this pest. However, studies are needed to assess the compatibility between these methods, aiming at their use in integrated management programs for this pest. The aim of this study was to evaluate the compatibility of the nematodes Steinernema carpocapsae ALL and Heterorhabditis sp. JPM4 with aqueous extracts prepared from dried plant of cinnamon leaf, twig and fruit (Melia azedarach), rue leaf (Ruta graveolens), ginger (Zingiber officinale) and garlic (Allium sativum) for the control of $C$. capitata. The bioassay was carried out in completely randomized design with four replicates per treatment. Each replication consisted of a glass tube containing $1 \mathrm{~mL}$ of plant extract $40 \% \mathrm{w} / \mathrm{v}$ and $1 \mathrm{~mL}$ suspension of entomopathogenic nematodes with $1800 \mathrm{JI} / \mathrm{mL}$ for S. carpocapsae ALL and $600 \mathrm{JI} / \mathrm{mL}$ for Heterorhabditis sp. JPM4. The viability and infectivity of this nematode were evaluated on C. capitata larvae after 48 and 120 hours. It was found that all extracts reduced the viability and infectivity of both nematodes and they were incompatible after 120 hours of exposure. The nematode Heterorhabditis sp. JPM4 was more sensitive than the $S$. carpocapsae ALL as it showed, in the first 48 hours, a reduction in the viability and infectivity of more than 80 and $75 \%$, respectively, when exposed to all the extracts except the ginger.
\end{abstract}

Key words: Steinernema carpocapsae, Heterorhabditis sp, Melia azedarac, Ruta graveolen, Allium sativu, Zingiber officinale

\section{Introdução}

O Brasil está entre os três maiores produtores mundiais de frutas, porém a sua capacidade de exportação ainda é baixa, devido às condições fitossanitárias do produto, o qual apresenta danos causados por pragas, principalmente, a moscadas-frutas Ceratitis capitata (Diptera: Tephritidae) (ZUCCHI, 2000).

A mosca-das-frutas, C. capitata, é a mais cosmopolita e invasora, sendo considerada a espécie que mais causa danos à fruticultura em todo o mundo (ZUCCHI, 2001). Essa praga é responsável por causar danos devido a oviposição das fêmeas e o consumo das larvas nos frutos, além do prejuízo causado pelas restrições impostas para comercialização do produto (WHITE; ÉLSONHARRIS, 1992; BUENO, 2000; MALAVASI et al., 2000).

Atualmente, o controle da mosca-das-frutas é realizado principalmente com produtos químicos. No entanto, essa forma de controle tem ocasionado impactos na saúde humana, no meio ambiente, além de favorecer o aparecimento de insetos resistentes e causar a morte de inimigos naturais. A preocupação com os diversos problemas causados pelo uso indiscriminado de produtos químicos tem conduzido à crescente busca por medidas eficazes de controle de pragas, que proporcionem um menor impacto ambiental e que sejam compatíveis com os programas de manejo integrado de pragas (NASCIMENTO; CARVALHO, 2000).

Nematóides entomopatogênicos, pertencentes às famílias Heterorhabditidae e Steinernematidae, são considerados excelentes agentes de controle biológico, demonstrando maior potencialidade em insetos-praga de solo e de ambientes crípticos. Estes entomopatógenos são ainda seletivos a um grande número de insetos não alvo, já que são restritos às pragas de solo, apresentam inocuidade ao meio ambiente e aos demais seres vivos, sendo um ótimo recurso a ser utilizado como componente do manejo integrado de pragas (GEORGIS et al., 2006; SHAPIRO-ILAN et al., 2006). Extratos vegetais também se destacam como importantes ferramentas em programas de manejo integrado de pragas, devido à eficiência e a facilidade de obtenção pelos produtores (MARTINS et al., 1995). 
Trabalhos foram desenvolvidos em condição de laboratório e campo para determinar a eficiência de diferentes espécies e isolados de nematóides entomopatogênicos sobre as diferentes fases de desenvolvimento de $C$. capitata, sendo observada elevada suscetibilidade deste inseto, especialmente do último instar larval e da fase de pupa, as quais encontram-se livres no solo (LINDEGREN; VAIL, 1986; LINDEGREN; WONG; MCINNIS, 1989; LINDEGREN, 1990; GAZIT; ROSSLER; GLAZER, 2000; LABORDA et al., 2003; ROHDE et al., 2010).

Em relação aos extratos vegetais, alguns trabalhos em condições de laboratório revelaram a ação inseticida de casca-de-limão Citrus limonia (Rutaceae), Cestrum parqui (Solanaceae), cinamomo Melia azedarach (Meliaceae), arruda Ruta graveolens (Rutaceae), gengibre Zingiber officinales (Zingiberaceae) e alho Allium sativum (Alliaceae) sobre as diferentes fases de desenvolvimento de $C$. capitata (SALVATORE et al., 2004; ZAPPATA et al., 2006; ROHDE, 2011).

O uso de nematóides entomopatogênicos e extratos vegetais pode ser promissor em programas de manejo integrado da mosca-das-frutas. A adoção destas duas formas de controle isoladas ou em mistura em uma mesma área pode melhorar a eficiência de controle da praga. Nesse sentido, Mahmoud (2007) observou em condições de laboratório que a associação do extrato de nim com o nematóide Steinernema feltiae melhorou o controle da mosca-das-frutas Bactrocera zonata (Diptera: Tephritidae).

No entanto, para a utilização integrada dessas formas de controle, é necessário conhecer o grau em que os nematóides podem ser afetados pelos extratos, determinando assim a compatibilidade entre as mesmas. Nesse sentido, Abdel-Rasek e Gowen (2002), Krishnayya e Grewal (2002) e Mahmoud (2007) verificaram que extratos de nim são compatíveis com diferentes nematóides entomopatogênicos. Por outro lado, Rovesti e
Deseo (1989) e Ramirez et al. (2009) observaram elevada toxicidade do extrato de nim e de mostarda, respectivamente, para os nematóides pertencentes aos gêneros Steinernema e Heterorhabditis.

Espécies de nematóides podem ser diferentes quanto à sensibilidade aos diversos extratos vegetais. Assim, o objetivo desse trabalho foi avaliar a compatibilidade de diferentes extratos vegetais com os nematóides Steinernema carpocapsae ALL e Heterorhabditis sp. JPM4, sendo que tanto os extratos quanto os nematóides selecionados tiveram sua ação inseticida sobre larvas no final do terceiro instar e pupas de $C$. capitata comprovada em testes preliminares.

\section{Material e Métodos}

\section{Criação de Ceratitis capitata}

Para o início da criação, foram utilizadas pupas provenientes do Centro de Energia Nuclear na Agricultura - CENA (Piracicaba, SP). A criação foi mantida em condições controladas de temperatura de $25 \pm 2{ }^{\circ} \mathrm{C}$, umidade relativa de $70 \pm 10 \%$ e fotofase de 12 horas, segundo metodologia proposta por Silva (1990).

\section{Obtenção dos nematóides entomopatogênicos}

Para a realização dos bioensaios, foram utilizados os isolados Heterorhabditis sp. JPM4 (originário de Minas Gerais, Brasil) e Steinernema carpocapsae ALL (originário da Carolina do Norte, EUA). Estes nematóides foram selecionados, pois de acordo com Rohde (2011) todos tiveram ação inseticida sobre larvas e pupas de C. capitata em condições de laboratório e casa-de-vegetação. A multiplicação dos nematóides foi realizada por meio do método in vivo, adaptado a partir de Woodring e Kaya (1988), utilizando-se lagartas de último ínstar de Galleria mellonella (Lepidoptera: Pyralidae). Foram utilizados juvenis infectivos de nematóides armazenados por no máximo 72 horas após a multiplicação, com viabilidade acima de 95\%. 


\section{Obtenção dos extratos vegetais}

Foram utilizados extratos aquosos preparados a partir de partes vegetais secas como a folha, o ramo ou o fruto de cinamomo Melia azedarach (Meliaceae), a folha de arruda Ruta graveolens (Rutaceae), a raíz do gengibre Zingiber officinales (Zingiberaceae) e o grão do alho Allium sativum (Alliaceae). Estes extratos foram selecionados, pois de acordo com Rohde (2011) todos tiveram ação inseticida, quando aplicados em condições de laboratório na concentração de $20 \%$, sobre larvas e pupas de C. capitata, com destaque para o extrato de cinamomo, o qual teve efeito também sobre a fase adulta. Para obtenção dos extratos, os vegetais foram mantidos em uma estufa a $90 \pm 2^{\circ} \mathrm{C}$, durante cinco dias, moídos em um triturador do tipo moinho de peneira de 17 mesh, e então diluídos em água destilada, na proporção de $40 \%$ p/v (peso das plantas secas/volume de água destilada) e deixados durantes 24 horas. Após esse período, as suspensões foram filtradas com o auxílio de um tecido do tipo "voil", para posterior teste de compatibilidade com nematóides entomopatogênicos.

Avaliação da compatibilidade de extratos vegetais com nematóides entomopatogênicos

Foi avaliada a compatibilidade dos extratos vegetais com os nematóides entomopatogênicos, de acordo com a metodologia modificada de Vainio (1992), a qual é utilizada para avaliar a compatibilidade entre nematóides e produtos fitossanitários. Esta metodologia foi adotada por não haver um protocolo padrão para estudo da compatibilidade de extratos vegetais com entomopatógenos. Os tratamentos foram: extratos aquosos preparados com vegetais secos da folha, ramo ou fruto de cinamomo, folha de arruda, gengibre, alho e água destilada (como testemunha) combinados com os nematóides $S$. carpocapsae ALL ou Heterorhabditis sp. JPM . Foram utilizadas quatro repetições por tratamento, cada uma constituída de um tubo de vidro contendo $1 \mathrm{~mL}$ de extrato vegetal $40 \% \mathrm{p} / \mathrm{v}$ e $1 \mathrm{~mL}$ de suspensão de nematóide entomopatogênico, com 1800 JI/ $\mathrm{mL}$ para $S$. carpocapsae ALL e $600 \mathrm{JI} / \mathrm{mL}$ para Heterorhabditis sp. JPM4. A concentração dos extratos vegetais de $40 \% \mathrm{p} / \mathrm{v}$ foi adotada, pois de acordo com a metodologia proposta por Vainio (1992) deve ser usado o dobro da concentração recomendada do produto. Como não existem recomendações de uso para o controle de $C$. capitata com extratos vegetais, usou-se como padrão, a concentração de $20 \%$, adotada no trabalho realizado por Rohde (2011), no qual foi verificada ação inseticida dos mesmos extratos vegetais usados no presente trabalho sobre larvas no final do último instar e pupas esta praga. Como tratamento testemunha foi adicionado apenas água destilada na suspensão de nematoides. O bioensaio foi conduzido em delineamento inteiramente casualizado, e os tubos foram mantidos em câmara climatizada $\left(25 \pm 2^{\circ} \mathrm{C}, 70 \pm 10 \%\right.$ de umidade, fosfatase de 12 horas). Foi avaliada a viabilidade e a infectividade dos nematóides após 48 e 120 horas. Para avaliação da viabilidade, de cada repetição, foram retiradas três alíquotas de $0,1 \mathrm{~mL}$ e contado o número de $\mathrm{JI}$ vivos e mortos, sendo considerados mortos aqueles que não responderam ao estímulo com estilete. Para avaliação da infectividade, os tubos foram completados com água destilada $(3 \mathrm{~mL})$ e deixados para decantar por meia hora em geladeira a $8^{\circ} \mathrm{C}$, o sobrenadante (cerca de $3 \mathrm{~mL}$ ) foi descartado e a lavagem repetida por três vezes para eliminação dos resíduos dos extratos. O mesmo processo foi realizado para o tratamento testemunha. Após a terceira lavagem, de cada repetição, foram retiradas três amostras de $1 \mathrm{~mL}$ e aplicadas em três placas de Petri contendo papel filtro e 10 larvas no final do terceiro instar de $C$. capitata. A concentração foi mantida em $60 \mathrm{JI} /$ inseto para $S$. carpocapsae ALL e 20J//inseto para Heterorhabditis sp. JPM4. As placas foram acondicionadas em potes plásticos e mantidas nas mesmas condições descritas anteriormente. Após cinco dias, as larvas mortas foram dissecadas, para confirmação da presença do nematóide. Os dados foram transformados 
em $\operatorname{arcseno} \sqrt{X / 100}$, submetidos à análise de variância (teste $\mathrm{F}$ ) e as médias comparadas entre si pelo teste de Scott - Knott $(\mathrm{P} \leq 0,05)$, com auxílio do programa Sisvar (FERREIRA, 2000). Os dados de mortalidade de nematóides foram corrigidos pela fórmula de Abbott (1925) e os dados de infectividade foram transformados para valores de porcentagem de redução de infectividade, em relação à testemunha. Valores de mortalidade de nematóides e de redução de infectividade maiores que $50 \%$ foram considerados incompatíveis, de acordo com a classificação proposta por Vainio (1992).

\section{Resultados e Discussão}

Todos os extratos estudados reduziram a viabilidade e a infectividade de S. carpocapsae ALL, diferindo do tratamento testemunha já nas primeiras 48 horas (Tabela 1). Nessa primeira avaliação, apenas os extratos de ramo de cinamomo e alho foram compatíveis com esse nematóide, causando redução de viabilidade de 22,8 e $25,2 \%$, respectivamente (Tabela 2). Em relação à infectividade do nematóide, o efeito dos extratos foi mais severo, causando redução de 56,0 a $80,0 \%$, sendo todos considerados incompatíveis (Tabela 3). Na segunda avaliação (após 120 horas), os efeitos negativos dos extratos sobre o nematóide foram intensificados, com redução na viabilidade e infectividade superior a 96,0 e $85,6 \%$, respectivamente, sendo todos considerados incompatíveis.

Tabela 1. Viabilidade e infectividade ( \pm EP) de Steinernema carpocapsae ALL após 48 e 120 horas de exposição aos extratos vegetais.

\begin{tabular}{ccccc}
\hline \multirow{2}{*}{ Tratamentos } & \multicolumn{2}{c}{48 horas $^{1,2}$} & \multicolumn{2}{c}{120 horas } \\
\cline { 2 - 5 } & Viabilidade & Infectividade & Viabilidade & Infectividade \\
\hline Testemunha & $83,71 \pm 0,99 \mathrm{a}$ & $83,30 \pm 1,35 \mathrm{a}$ & $84,85 \pm 2,81 \mathrm{a}$ & $51,63 \pm 2,15 \mathrm{a}$ \\
Fruto de cinamomo & $30,23 \pm 3,50 \mathrm{c}$ & $25,83 \pm 2,50 \mathrm{c}$ & $0,77 \pm 0,77 \mathrm{~b}$ & $0,83 \pm 0,83 \mathrm{c}$ \\
Folha de cinamomo & $37,98 \pm 4,74 \mathrm{c}$ & $36,63 \pm 4,09 \mathrm{~b}$ & $1,66 \pm 0,98 \mathrm{~b}$ & $1,65 \pm 1,65 \mathrm{c}$ \\
Ramo de cinamomo & $64,61 \pm 3,45 \mathrm{~b}$ & $34,15 \pm 2,49 \mathrm{~b}$ & $7,45 \pm 0,85 \mathrm{~b}$ & $0,00 \pm 0,00 \mathrm{~b}$ \\
Gengibre & $37,43 \pm 2,87 \mathrm{c}$ & $24,13 \pm 2,86 \mathrm{c}$ & $3,37 \pm 2,16 \mathrm{~b}$ & $3,33 \pm 3,33 \mathrm{~b}$ \\
Alho & $63,44 \pm 3,26 \mathrm{~b}$ & $21,63 \pm 3,18 \mathrm{c}$ & $5,55 \pm 2,07 \mathrm{~b}$ & $3,30 \pm 0,00 \mathrm{~b}$ \\
Arruda & $16,56 \pm 1,71 \mathrm{~d}$ & $16,63 \pm 1,37 \mathrm{c}$ & $2,64 \pm 1,62 \mathrm{~b}$ & $9,98 \pm 6,81 \mathrm{~b}$ \\
\hline $\mathrm{CV}(\%)$ & 7,63 & 9,26 & 49,89 & 54,62 \\
\hline
\end{tabular}

${ }^{1}$ Médias seguidas da mesma letra na mesma coluna não diferem entre si pelo teste Scott-Knott $(\mathrm{P} \leq 0,05 \%) .{ }^{2} \mathrm{Dados}$ originais apresentados, transformados em $\operatorname{arcsen} \sqrt{x / 100}$ para análise.

Fonte: Elaboração dos autores.

Tabela 2. Mortalidade corrigida (\%) de Steinernema carpocapsae ALL, após 48 e 120 horas de exposição aos extratos vegetais e classificação de compatibilidade.

\begin{tabular}{ccccc}
\hline \multirow{2}{*}{ Tratamentos } & \multicolumn{2}{c}{48 horas } & \multicolumn{2}{c}{ 120 horas } \\
\cline { 2 - 5 } & Mortalidade corrigida $^{1}$ & Classificação $^{2}$ & Mortalidade corrigida $^{1}$ & Classificação $^{2}$ \\
\hline Fruto de cinamomo & 63,89 & Incompatível & 99,09 & Incompatível \\
Folha de cinamomo & 54,63 & Incompatível & 98,04 & Incompatível \\
Ramo de cinamomo & 22,82 & Compatível & 100,00 & Incompatível \\
Gengibre & 55,29 & Incompatível & 96,03 & Incompatível \\
Alho & 25,21 & Compatível & 93,46 & Incompatível \\
Arruda & 80,22 & Incompatível & 96,86 & Incompatível \\
\hline
\end{tabular}

${ }^{1}$ Mortalidade corrigida pela fórmula de Abbott (1925). ${ }^{2}$ Classificação de compatibilidade segundo Vainio (1992).

Fonte: Elaboração dos autores. 
Tabela 3. Redução de infectividade (\%) de Steinernema carpocapsae ALL, após 48 e 120 horas de exposição aos extratos vegetais e classificação de compatibilidade dos extratos vegetais com o nematóide em relação à infectividade sobre larvas de Ceratitis capitata.

\begin{tabular}{|c|c|c|c|c|}
\hline \multirow[b]{2}{*}{ Tratamentos } & \multicolumn{2}{|c|}{48 horas } & \multicolumn{2}{|c|}{120 horas } \\
\hline & $\begin{array}{c}\text { \% redução } \\
\text { infectividade }\end{array}$ & Classificação ${ }^{2}$ & $\begin{array}{c}\text { \% redução } \\
\text { infectividade }\end{array}$ & Classificação² \\
\hline $\begin{array}{l}\text { Fruto de } \\
\text { cinamomo }\end{array}$ & 69,00 & Incompatível & 98,39 & Incompatível \\
\hline $\begin{array}{l}\text { Folha de } \\
\text { cinamomo }\end{array}$ & 56,03 & Incompatível & 96,80 & Incompatível \\
\hline $\begin{array}{l}\text { Ramo de } \\
\text { cinamomo }\end{array}$ & 59,00 & Incompatível & 85,60 & Incompatível \\
\hline Gengibre & 71,04 & Incompatível & 93,50 & Incompatível \\
\hline Alho & 74,04 & Incompatível & 93,60 & Incompatível \\
\hline Arruda & 80,04 & Incompatível & 80,70 & Incompatível \\
\hline
\end{tabular}

${ }^{1}$ Porcentagem de redução de infectividade em relação à testemunha. ${ }^{2}$ Classificação de compatibilidade segundo Vainio (1992).

Fonte: Elaboração dos autores.

Já o isolado Heterorhabditis sp. JPM4 foi compatível com esse nematóide (Tabelas 4 e 5). mais sensível em relação ao S. carpocapsae ALL, Ao analisar a infectividade, todos os extratos foram pois apresentou na primeira avaliação (48 horas) incompatíveis, pois causaram redução superior redução na viabilidade superior a 80,0\% quando a 75,4\% (Tabela 6). Na segunda avaliação (120 exposto a todos os extratos, exceto o de gengibre, horas), todos os extratos causaram $100 \%$ de redução o qual causou redução de $45,5 \%$, sendo o único na viabilidade e infectividade do nematóide, sendo considerados incompatíveis.

Tabela 4. Viabilidade e infectividade ( \pm EP) de Heterorhabditis sp. JPM4, após 48 e 120 horas de exposição aos extratos vegetais.

\begin{tabular}{ccccc}
\hline \multirow{2}{*}{ Tratamentos } & \multicolumn{2}{c}{48 horas $^{1,2}$} & \multicolumn{2}{c}{120 horas $^{1,2}$} \\
\cline { 2 - 5 } & Viabilidade & Viabilidade & Infectividade \\
\hline $\begin{array}{c}\text { Testemunha } \\
\text { Fruto de }\end{array}$ & $92,46 \pm 2,81 \mathrm{a}$ & $50,80 \pm 2,09 \mathrm{a}$ & $89,37 \pm 2,69 \mathrm{a}$ & $34,13 \pm 1,58 \mathrm{a}$ \\
cinamomo & $3,07 \pm 1,82 \mathrm{~d}$ & $4,00 \pm 3,15 \mathrm{~b}$ & $0,00 \pm 0,00 \mathrm{~b}$ & $0,00 \pm 0,00 \mathrm{~b}$ \\
$\begin{array}{c}\text { Folha de } \\
\text { cinamomo }\end{array}$ & $0,00 \pm 0,00 \mathrm{e}$ & $0,00 \pm 2,09 \mathrm{~b}$ & $0,00 \pm 0,00 \mathrm{~b}$ & $0,00 \pm 0,00 \mathrm{~b}$ \\
$\quad \begin{array}{c}\text { Ramo de } \\
\text { cinamomo }\end{array}$ & $0,64 \pm 0,64 \mathrm{e}$ & $6,63 \pm 1,37 \mathrm{~b}$ & $0,00 \pm 0,00 \mathrm{~b}$ & $0,00 \pm 0,00 \mathrm{~b}$ \\
Gengibre & $50,39 \pm 2,58 \mathrm{~b}$ & $4,15 \pm 2,10 \mathrm{~b}$ & $0,00 \pm 0,00 \mathrm{~b}$ & $0,00 \pm 0,00 \mathrm{~b}$ \\
Alho & $18,44 \pm 2,65 \mathrm{c}$ & $8,77 \pm 2,84 \mathrm{~b}$ & $0,00 \pm 0,00 \mathrm{~b}$ & $0,00 \pm 0,00 \mathrm{~b}$ \\
Arruda & $0,00 \pm 0,00 \mathrm{e}$ & $0,00 \pm 1,58 \mathrm{~b}$ & $0,00 \pm 0,00 \mathrm{~b}$ & $0,00 \pm 0,00 \mathrm{~b}$ \\
\hline $\mathrm{CV}(\%)$ & 21,25 & 35,75 & 7,97 & 12,41 \\
\hline
\end{tabular}

${ }^{1}$ Médias seguidas da mesma letra na mesma coluna não diferem entre si pelo teste Scott-Knott $(\mathrm{P} \leq 0,05 \%) .{ }^{2} \mathrm{Dados}$ originais apresentados, transformados em $\operatorname{arcsen} \sqrt{x / 100}$ para análise.

Fonte: Elaboração dos autores. 
Tabela 5. Mortalidade corrigida (\%) de Heterorhabditis sp. JPM4, após 48 e 120 horas de exposição aos extratos vegetais e classificação de compatibilidade.

\begin{tabular}{|c|c|c|c|c|}
\hline \multirow[b]{2}{*}{ Tratamentos } & \multicolumn{2}{|c|}{48 horas } & \multicolumn{2}{|c|}{120 horas } \\
\hline & $\begin{array}{l}\text { Mortalidade } \\
\text { corrigida }^{1}\end{array}$ & Classificação ${ }^{2}$ & $\begin{array}{l}\text { Mortalidade } \\
\text { corrigida }^{1}\end{array}$ & Classificação $^{2}$ \\
\hline $\begin{array}{l}\text { Fruto de } \\
\text { cinamomo }\end{array}$ & 96,68 & Incompatível & 100,00 & Incompatível \\
\hline $\begin{array}{l}\text { Folha de } \\
\text { cinamomo }\end{array}$ & 100,00 & Incompatível & 100,00 & Incompatível \\
\hline $\begin{array}{l}\text { Ramo de } \\
\text { cinamomo }\end{array}$ & 99,31 & Incompatível & 100,00 & Incompatível \\
\hline Gengibre & 45,50 & Compatível & 100,00 & Incompatível \\
\hline Alho & 80,06 & Incompatível & 100,00 & Incompatível \\
\hline Arruda & 100,00 & Incompatível & 100,00 & Incompatível \\
\hline
\end{tabular}

${ }^{1}$ Mortalidade corrigida pela fórmula de Abbott (1925). ${ }^{2}$ Classificação de compatibilidade segundo Vainio (1992).

Fonte: Elaboração dos autores.

Tabela 6. Redução de infectividade (\%) de Heterorhabditis sp. JPM4, após 48 e 120 horas de exposição à diferentes extratos vegetais e classificação de compatibilidade dos extratos vegetais com o nematóide em relação à infectividade sobre larvas de Ceratitis capitata.

\begin{tabular}{ccccc}
\hline \multirow{2}{*}{ Tratamentos } & \multicolumn{2}{c}{48 horas } & \multicolumn{2}{c}{ 120 horas $^{\text {\% redução }}$} \\
\cline { 2 - 5 } infectividade $^{1}$ & Classificação $^{2}$ & $\begin{array}{c}\text { \% redução } \\
\text { infectividade }^{1}\end{array}$ & Classificação $^{2}$ \\
\hline $\begin{array}{c}\text { Fruto de } \\
\text { cinamomo } \\
\text { Folha de } \\
\text { cinamomo }\end{array}$ & 91,83 & Incompatível & 100,00 & Incompatível $^{\text {Ramo de }}$ \\
$\begin{array}{c}\text { cinamomo } \\
\text { Gengibre }\end{array}$ & 88,58 & Incompatível & 100,00 & Incompatível \\
Alho & 86,96 & Incompatível & 100,00 & Incompatível \\
Arruda & 81,83 & Incompatível & 100,00 & Incompatível \\
& 82,04 & Incompatível & 100,00 & Incompatível \\
Incompatível & 100,00 & Incompatível \\
\hline
\end{tabular}

${ }^{1}$ Porcentagem de redução de infectividade em relação à testemunha. ${ }^{2}$ Classificação de compatibilidade segundo Vainio (1992).

Fonte: Elaboração dos autores.

Ramirezetal. (2009) estudando a compatibilidade de extratos de mostarda, com os diferentes espécies de nematóides entomopatogênicos, pertencentes aos gêneros Steinernema e Heterorhabditis, também verificaram maior sensibilidade de Heterorhabditis sp. De acordo com Zasada e Ferris (2004), a maior sensibilidade de algumas espécies de nematóide ocorre devido às variações nas características biológicas das mesmas, tornando-as inerentemente mais ou menos resistentes aos efeitos nocivos de determinados produtos.
O mecanismo de ação dos extratos estudados sobre os nematóides ainda não está bem determinado. No entanto, sabe-se que o efeito nematicida de muitos extratos vegetais está relacionado à presença de substâncias produzidas pelo metabolismo secundário da planta, as quais podem danificar irreversivelmente determinados processos fisiológicos essenciais de certos organismos.

Nesse sentido, o efeito nematicida do extrato de cinamomo pode estar relacionado à presença de 
substâncias comuns, entre as espécies da família Meliaceae, tais como a azadiractina, meliantrol e salanina (MARTINEZ; VAN EMDEN, 2001). Já a ação do extrato de alho, sobre os nematóides, pode ser devido à presença de compostos sulfurosos, isotiocianatos, nitrilas, tiocianatos e epinitrilas na planta, enquanto que o extrato de arruda apresenta alcalóides (rutalinium, rutalidinam, rutacridona e rutalinidina), cumarinas (chalepeusina e gravileferona), flavonóides (rutina e hesperedina) e terpenos na planta, os quais apresentam atividade nematicida (SASANELLI, 1992; MARTINS et al., 1995). Para o extrato de gengibre, são necessários estudos bioquímicos da planta para determinar quais compostos podem atuar sobre os nematóides, reduzindo a sua viabilidade e infectividade.

Não foram encontrados trabalhos avaliando o efeito dos extratos vegetais estudados no presente trabalho sobre nematóides entomopatogênicos. No entanto, o efeito nematicida deles já foi registrado para alguns nematóides fitopatogênicos, tais como Meloidogyne javanica, M. arenaria, M. hapla, M. incognita, M. exigua, Scutellonema bradys, Tylenchulus semipenetrans, Aphelenchoides sacchari, Xiphinema index, Heterodera schachtii (SASANELLI, 1992; SASANELLI; D'ADDABOO, 1992; DIAS et al., 2000; AMARAL et al., 2002; COIMBRA et al., 2006; SALGADO; CAMPOS, 2003; GARDIANO et al., 2009).

Em trabalhos semelhantes, Abdel-Rasek e Gowen (2002) e Mahmoud (2007) observaram que a combinação de nematóides entomopatogênico pertencentes aos gêneros Steinernema e Heterohabditis com extrato de plantas de nim pode ser favorável para o controle de Plutella xylostella (Lepidoptera: Yponomeutidae) e Bactrocera zonata (Diptera: Tephritidae), respectivamente, indicando a compatibilidade entre os dois métodos de controle. Os autores concluíram ainda que a associação dos dois métodos pode incrementar o controle desses insetos-praga.
Por outro lado, Stark (1996) observou que o produto Margosan-O, uma formulação comercial do nim, foi incompatível com os nematóides $S$. carpocapsae, S. feltiae e S. glaseri. Rovesti e Deseo (1989) verificaram que extrato aquoso de nim também foi tóxico para $S$. carpocapsae. Esses autores ressaltaram ainda que a atividade dos extratos vegetais sobre os nematóides entomopatogênicos é influenciada de acordo com a idade da planta, parte do vegetal utilizada e método de extração. Assim, os extratos aqui estudados podem apresentar menor toxidade para S. carpocapsae ALL e Heterorhabditis sp. JPM4, quando preparados de maneira diferente ou em concentrações menores.

Com os resultados do presente trabalho, pode-se concluir que os extratos de folha, ramo e fruto de cinamomo, arruda, gengibre e alho na concentração de $40 \%$ p/v são incompatíveis com $S$. carpocapsae ALL e Heterorhabditis sp. JPM4. Vale ressaltar, no entanto, que houve exposição máxima dos extratos vegetais sobre os nematóides entomopatogênicos, sendo necessários novos estudos para avaliar o efeito de concentrações mais baixas.

\section{Referências}

ABBOTT, W. S. A method of computing the effectiveness of an insecticide. Journal of Economic Entomology, College Park, v. 18, p. 265-266, 1925.

ABDEL-RASEK, A. S.; GOWEN, S. The integrated effect to the nematode-bacteria complex and neem plant extracts against Plutella xylostella (L.) larvae (Lepidoptera: Yponomeutidae) on chinese cabbage. Archive Phytopathology, Berlin, v. 35, n. 2, p. 181-188, 2002.

AMARAL, D. R.; OLIVEIRA, D. F.; CAMPOS, V. P.; CARVALHO, D. A. Efeito de alguns extratos vegetais na eclosão, mobilidade, mortalidade e patogenicidade de Meloidogyne exigua do cafeeiro. Nematologia Brasileira, Campinas, v. 26, n. 1, p. 43-48, 2002.

BUENO, L. N. Las moscas de las frutas: importancia económica, aspectos taxonómicos, distribución mundial de los géneros de importancia económica. In: SEMINARIO TALLER SOBRE EL MANEJO DE LAS 
MOSCAS DE LAS FRUTAS EN EL DEPARTAMENTO DE ARAUCA, 1., 2000, Saravena, Colombia. Primer... Saravena, Colômbia: [s.n], 2000. p. 1-19.

COIMBRA, J. L.; SOARES, A. C. F.; GARRIDO, M. S.; SOUZA, C. S.; RIBEIRO, F. L. B. Toxicidade de extratos vegetais a Scutellonema bradys. Pesquisa Agropecuária Brasileira, Brasília, v. 41, n. 7, p. 1209-1211, 2006.

DIAS, C. R.; SCHWAN, A.V.; EZEQUIEL, D. P.; SARMENTO, M. C.; FERRAZ, S. Efeito de extratos aquosos de plantas medicinais na sobrevivência de juvenis de Meloidogyne incognita. Nematologia Brasileira, Campinas, v. 24, n. 2, p. 203-210, 2000.

FERREIRA, D. F. Análise estatística por meio do SISVAR (sistema para análise de variância) para Windows versão 4.0. In: REUNIÃO ANUAL DA REGIÃO BRASILEIRA DA SOCIEDADE INTERNACIONAL DE BIOMETRIA, 1., 2000, São Carlos. Anais... São Carlos: UFSCar, 2000. p. 255-258.

GARDIANO, C. G.; FERRAZ, S.; LOPES, E. A.; FERREIRA, P. A.; AMORA, D. X.; FREITAS, L. G. Avaliação de extratos aquosos de várias espécies vegetais, aplicados ao solo, sobre Meloidogyne javanica (Treub, 1885) Chitwood, 1949. Semina: Ciências Agrárias, Londrina, v. 30, n. 3, p. 551-556, 2009.

GAZIT, Y.; ROSSLER, Y.; GLAZER, I. Evaluation of entomopathogenic nematodes for the control of mediterranean fruit fly (Diptera: Tephritidae). Biocontrol Science and Technology, Abingdon, v. 10, n. 2, p. 157164, 2000.

GEORGIS, R.; KOPPENHÖFER, A. M.; LACEY, L. A.; BÉLAIR, G.; DUNCAN, L. W.; GREWAL, P. S.; SAMISH, M.; TAN, L.; TORR, P.; van TOL, R.W.H.M. Successes and failures in the use of parasitic nematodes for pest control. Biological Control, San Diego, v. 38, n. 1, p. 103-123, 2006.

KRISHNAYYA, P. V.; GREWAL, P. S. Effect of neem and selected fungicides on viability and virulence of the entomopathogenic nematode Steinernema feltiae. Biocontrol Science and Technology, Abingdon, v. 12, n. 3, p. 259-266, 2002.

LABORDA, R.; BARGUES, L.; NAVARRO, C.; BARAJAS, O.; ARROYO, M.; GARCIA, E. M., MONTORO, E.; LLOPIS, E.; MARTINEZ, A.; SAYAGUES, J. M. Susceptibility of the mediterranean fruit fly (Ceratitis capitata) to entomopathogenic nematode Steinerma spp. ("Biorend C"). Bulletin OILB/ SROP, Dijon, v. 26, n. 6, p. 95-97, 2003.

LINDEGREN, J. E. Field suppression of three fruit fly species (Diptera: Tephritidae) with Steinernema carpocapsae. In: INTERNATIONAL COLLOQUIUM
ON INVERTEBRATE MICROBIAL CONTROL, 5., 1990, Foz de Iguaçu. Proceedings... Foz de Iguaçu: [s.n], 1990. p. 223. Abstracts.

LINDEGREN, J. E.; VAIL, P. V. Susceptibility of mediterranean fruit fly, melon fly, and oriental fruit fly (Diptera: Tephritidae) to the entomogenous nematode Steinernema feltiae in laboratory tests. Environmental Entomology, Lanham, v. 15, n. 3, p. 465-468, 1986.

LINDEGREN, J. E.; WONG, T. T.; MCINNIS, D. O. Response of mediterranean fruit fly (Diptera: Tephritidae) to the entomogenous nematode Steinernema feltiae in field tests in Hawaii. Environmental Entomology, Lanham, v. 19, n. 2, p. 383-386, 1989.

MAHMOUD, F. Combining the botanical insecticides nsk extract, neemazal t 5\%, neemix $4.5 \%$ and the entomopathogenic nematode Steinernema feltiae cross n 33 to control the peach fruit fly, Bactrocera zonata (Saunders). Plant Protection Science, Praha, v. 43, n. 1, p. 19-25, 2007.

MALAVASI, A.; ZUCCHI, R. A.; SUGAYAMA, R. L. Biogeografia. In: MALAVASI, A.; ZUCCHI, R. A. Moscas-das-frutas de importância econômica no Brasil: conhecimento básico e aplicado. Ribeirão Preto: Holos, 2000. p. 93-98.

MARTINEZ, S. S.; VAN EMDEN, H. F. Growth disruption, abnormalities and mortality of Spodoptera littoralis caused by azadirachtin. Neotropical Entomology, Londrina, v. 30, n. 1, p. 113-125, 2001.

MARTINS, E. R.; CASTRO, D. M.; CASTELLANI, D. C.; DAS, J. E. Plantas medicinais. Viçosa: Imprensa Universitária, $1995.25 \mathrm{p}$.

NASCIMENTO, A. S.; CARVALHO, R. S. Manejo integrado de mosca-das-frutas. In: MALAVASI, A.; ZUCCHI, R. A. Moscas-das-frutas de importância econômica no Brasil: conhecimento básico e aplicado. Ribeirão Preto: Holos, 2000. p. 169-174.

RAMIREZ, R. A.; HENDERSON, D. R.; RIGA, E.; LACEY, L. A.; SNYDER, W. Harmful effects of mustard bio-fumigants on entomopathogenic nematodes. Biological Control, San Diego, v. 48, n. 2, p. 147-154, 2009.

ROHDE, C. Avaliação de nematoides entomopatogênicos e extratos vegetais aquosos para o controle da moscadas-frutas Ceratitis capitata (Wiedemann) (Diptera: Tephritidae). 2011. Tese (Doutorado em Entomologia) Universidade Federal de Lavras, Lavras.

ROHDE, C.; MOINO JUNIOR, A.; SILVA, M. A. T.; CARVALHO, F. D.; FERREIRA, C. S. Influence of soil temperature and moisture on the infectivity of entomopathogenic nematodes (Rhabditida: 
Heterorhabiditidae e Steinernematidae) against larvae of Ceratitis capitata (Wiedemann) (Diptera: Tephritidae). Neotropical Entomology, Londrina, v. 39, n. 4, p. 608$611,2010$.

ROVESTI, L.; DESEO, K. K. Effect of neem kernel extract on Steinernematid and Heterorhabditid nematodes. Nematologica, Leiden, v. 35, n. 5, p. 493496, 1989.

SALGADO, S. M.; CAMPOS, V. Eclosão e mortalidade de Meloidogyne exigua em extratos e em produtos naturais. Fitopatologia Brasileira, Brasília, v. 28, n. 2, p. 166-170, 2003.

SALVATORE, A.; BORKOSKY, S.; WILLINK, E.; BARDÓN, A. Toxic effects of lemon peel constituents on Ceratitis capitata. Journal of Chemical Ecology, New York, v. 30, n. 2, p. 323-333, feb. 2004.

SASANELLI, N. Nematicidal activity of aqueous extracts from leaves of Ruta graveolens on Xiphinema index. Nematologia Mediterrânea, Bari, v. 20, n. 1, p. 53-55, 1992.

SASANELLI, N.; D'ADDABBO, T. D. Effect of Cineraria maritima, Ruta graveolens and Tagetes erecta on the hatching of Heterodera schachtii. Nematologia Mediterrânea, Bari, v. 20, n. 1, p. 59-51, 1992.

SHAPIRO-ILAN, D. I.; GOUGE, D. H.; PIGGOTT, S. J.; FIFE, J. P. Applications technology and environmental considerations for use of entompathogenic nematodes in biological control. Biological Control, San Diego, v. 38, n. 1, p. 124-133, 2006.

SILVA, A. C. Efeito inseticida, deterrente e suppressor alimentar de alguns extratos vegetais sobre Ceratitis capitata (Wiedemann, 1824) (Diptera: Tephritidae) e Ascia monuste orseis (Latreille, 1819) (Lepidoptera: Pieridae) em laboratório. 1990. Tese (Doutorado em Entomologia) - Universidade Federal de Lavras, Lavras.
STARK, J. D. Entomopathogenic nematodes (Rhabditida: Steinernematidae): toxicity of neem. Journal of Economic Entomology, Lanham, v. 89, n. 1, p. 68-73, 1996.

VAINIO, A. Guideline for laboratory testing of the side-effects of pesticides on entomophagous nematodes Steinernema spp. IOBC/WPRS Bulletin, Montfavet, v. 15, n. 2, p. 145-147, 1992.

WHITE, I. A.; ELSON-HARRIS, M. M. Introduction. In: WHITE, I. A.; ELSON-HARRIS, M. M. Fruit flies of economic significance: their identification and bionomics. Australia: CAB International, 1992. p. 1-14.

WOODRING, J. L.; KAYA, H. K. Steinernematidae and Heterorhabditidae nematodes: a handbook of techniques soulhern cooperative. Arkansas: Arkansas Agricultural Experiment Station Falleteville, 1988. (Series Bulletin).

ZAPPATA, N.; BUDIA, F.; VINUELA, E.; MEDINA, P. Insecticidal effects of various concentrations of selected extractions of Cestrum parqui on adult and immature Ceratitis capitata. Journal of Economic Entomology, College Park, v. 99, n. 2, p. 359-365, 2006.

ZASADA, I. A.; FERRIS, H. Nematode suppression with brassicaceous amendments: application based upon glucosinolate profiles. Soil Biology and Biochemistry, v. 36, n. 7, p. 1017-1024, 2004.

ZUCCHI, R. A. Mosca-do-mediterrâneo, Ceratitis capitata (Diptera: Tephritidae). In: VILELA, E. F.; ZUCCHI, R. A.; CANTOR, F. Histórico e impacto das pragas introduzidas no Brasil. Ribeirão Preto: Holos, 2001. p. 15-22.

A. Taxonomia. In: MALAVASI, A.; ZUCCHI, R. A. Moscas-das-frutas de importância econômica no Brasil: conhecimento básico e aplicado. Ribeirão Preto: Holos, 2000. p. 13-24. 